\title{
Culturally Diverse Discharge Planning and Education
}

\author{
Dulce Santacroce DNP, RN, CCM* \\ RN-BSN Coordinator, Touro University Nevada, USA
}

*Corresponding author: Dulce Santacroce, RN-BSN Coordinator, Touro University Nevada, USA.

Received Date: January 24, 2019

Published Date: February 20, 2019

\begin{abstract}
Readmission rates have been increasing over the past several years; therefore, the Affordable Care Act has required Social Security to create a program known as the Hospital Readmissions Reduction Program (HRRP). The main objective of the HRRP is for the Centers for Medicare and Medicaid Services (CMS) to reduce reimbursement to hospitals with excessive readmission rates. Extensive research has revealed several factors that are contributing to an increase in readmission rates and poor patient outcomes; one is a gap in communication and lack of cultural competency in nursing care. Another contribution to this phenomenon is poorly managed transitions of patients with chronic diseases from the hospital into the community due to inadequate discharge planning. Therefore, the aim of this project will be to encourage nursing faculty to develop curriculum for culturally diverse discharge planning and education for all levels of nursing courses, with the goal being to contribute to improved patient outcomes and decreased readmission rates among the various cultures. Utilizing Madeleine Leininger's Theory of Culture Care and Universality as a model, a conceptual framework Culturally Diverse Discharge Planning and Education was created to assist in developing a curriculum to increase awareness and foster student nurse critical thinking skills of the discharge planning and education process. Instructors of various local Registered Nursing programs will be invited to participate in a "train the trainer" type presentation of curriculum to introduce cultural diversity for discharge planning and education into the classroom, which will be developed and delivered by using Power Point, case studies, student collaboration, and role play. Instructors will be asked at the end of the presentation to provide their opinions of the value of this curriculum and the chance they will incorporate cultural discharge planning and education into their curriculum.
\end{abstract}

\section{Introduction and Background}

Hospitals have not been accountable for the transition of safe patient care after discharge. This phenomenon has created poor patient outcomes, increased readmission rates, and increased healthcare costs. Therefore, the Affordable Care Act (ACA) has required Social Security to create a program known as the Hospital Readmissions Reduction Program (HRRP) [1]. The main objective of the HRRP is for the Centers for Medicare and Medicaid Services (CMS) to reduce reimbursement to hospitals with excessive readmission rates. Readmission has been defined as admission within 30 days of discharge (Centers for Medicare \& Medicaid Services [CMS], 2014). Research has revealed a gap in communication during nursing care as one of the main reasons for the increase in readmissions [2]. The Center for Healthcare Quality and Payment Reform (CHQPR) (2013) has discovered, through extensive research, other reasons for readmissions include poorly managed transitions of patients with chronic diseases from the hospital into the community due to lack of comprehension of diverse cultures causing inadequate discharge planning.
According to the Centers for Disease Control and Prevention (CDC, 2015) the total national healthcare expenditures for 2013 in the United States was $\$ 2.9$ trillion. Medicare revealed hospital readmissions after 30 days of discharge were the primary cause for increased hospital costs with projections that will surpass $\$ 5$ trillion in 2015 [3]. Therefore, CMS has imposed strict penalties and reductions to hospital reimbursements. As health care costs continue to escalate, the ACA has demanded hospitals take responsibility for improving discharge planning, which should start the first day of admission.

Lack of communication among health care professionals is a systemic issue in acute and sub-acute facilities [4]. Several causes have been identified, and include the breakdown in communication, post-discharge care that is inadequate, poor follow-up care, and patients discharged prematurely [4]. Resolution of this phenomenon needs to include proper communication among interdisciplinary team members that also include the patient their family, and cultural needs to provide positive outcomes and reduce 
readmission rates. Culturally Diverse Discharge Planning and Education was a practice change project.

\section{Review of the Literature}

A thorough search of digital databases through Touro University Nevada and Walden Alumni libraries was conducted using the following search words, hospital readmissions, discharge planning, discharge education and planning, communication breakdown in healthcare, poor patient outcomes, patient and family centered discharge, cultural diversity and discharge education, nursing handoffs, and ISBAR.

\section{Method}

\section{Conceptual framework}

A conceptual framework was developed from various orientational terms of Leininger's Theory of Cultural Care and Universality. Madeleine Leininger is the leader in the field of transcultural nursing [5]. The center of the concept framework represents the patient and the family. The student nurse will be guided to self-reflect to bring awareness of their own beliefs and values. Through this exercise they will gain the knowledge to develop the skills necessary to provide culturally competent care for safe quality care and potentially reduce readmission rates. This education will also assist the nursing student to become aware of the cultural differences of their patient and family so they can provide the appropriate resources and education for a safe discharge into the community. Communication is priority; this will include the orientational terms, culture, and diversity. First included into care; is an inquiry of the patient's culture, which allows the patient to share religion and spirituality. Second included care; the care anticipated to improve health. Third included culture; the transmitted values, beliefs, and norms that guide patient decisions, motivations, and actions for treatment. Fourth included environmental factors; such as influences within one's geographic or ecological living area. The fifth included culture care; subjectively and objectively learned ways of life that enable another individual to sustain their wellbeing and health. The sixth included educational factors; formal or informal modes of learning to disseminate the treatment plan. It is important to include the learning style of the patient. If the patient requires a variety of activities, the use of pictures may benefit the patient in comprehending the knowledge necessary to promote quality health and reduce readmission rates [6].

Literature reveals poor patient outcomes and readmission rates are a phenomenon that is persistent. Therefore, this project facilitator invited faculty of local nursing programs to attend a presentation disseminating evidence pertaining to increased readmission rates in acute care settings due to staff nurses' lack of communication, cultural competence, and knowledge of the discharge process. A PowerPoint presentation was presented along with handouts of case studies. The handouts provided pertained to various cultures, values, and beliefs regarding healthcare. Participants were divided into groups provided computers to research disease processes and cultures of their patients, which also promoted collaboration and teamwork. They then roleplayed their scenario for the other groups to observe. There was a short debrief session for participants to provide feedback on their observations. At this point, the project facilitator was able to perform a formative evaluation to determine if the participants comprehend the concept of this project. At the end of this session, a discussion was opened to provide feedback as to the possibility of the participants incorporating this project into their courses.

\section{Population of interest}

The participants for this project were faculty members of local Associate Degree in Nursing (ADN) and Baccalaureate of Science Nursing (BSN) programs. Faculty members invited to the presentation were representative of every level of nursing programs. There were approximately four to six nursing school programs invited to participate in the project. Instructors facilitating the different levels of the nursing programs provided their opinion of the curriculum. Due to the diverse population in the Las Vegas and Henderson area this also provided various opinions representing diversity toward the curriculum developed.

\section{Setting}

The setting for this DNP project was located in a classroom at Touro University Nevada. The classroom chosen for the delivery of the presentation was dependent upon the number of replies received from the faculty members invited.

\section{Tools}

An interactive presentation was developed utilizing PowerPoint to disseminate pertinent information pertaining to culturally diverse discharge planning and education. The job descriptions and responsibilities of case managers and social workers was discussed. Participants were placed in groups for a team-based learning activity. Handout packets were distributed to participants containing objectives and outcomes of the DNP project. Within the packet, there was approximately three to four different case studies of patients of diverse cultures and disease processes to assess and discuss within their groups. The most common demographic ethnic and cultural groups were utilized for this presentation. Specifically, the case studies consisted of the Asian, Hispanic, African American, and illiterate populations, which are the most prevalent in the southwest area of the United States. Participants were provided approximately 20 minutes to collaborate and discuss the case study, they were asked to role-play the scenario they had decided on for the discharge process for their specific patient. Case studies and socio-drama provide the students with real-life situations that apply didactic content and theory to both the simulation and reallife situations. After each scenario, there was a short debriefing session.

The model "Train the Trainer" will be utilized for the presentation [7]. This model is a workshop that enables an experienced instructor to present information effectively through a specific training session to other instructors for the use of disseminating information to students [7]. The instructor will respond to participant questions and lead activities. This workshop provided the participants with valuable information and skills to deliver this curriculum themselves. Participants were asked 
to participate in this interactive presentation as students. An educational curriculum consisting of culturally diverse discharge education was incorporated into a Fundamental Nursing course. TUN's mission and values was incorporated into the curriculum along with keeping within compliance of the Quality and Safety Education for Nursing Competencies (QSEN) and the BSN Essentials. Participants posed as first semester Fundamental Nursing students of Touro University Nevada's Baccalaureate of Science Nursing program. Participants learned about the members of an interdisciplinary team and their responsibilities.

\section{Sample case study}

Participants worked in small groups; each group was provided a case study focused on a specific population.

A 73-year-old Asian Female K. F. was brought to the emergency room by her daughter stating her mother was discharged from the hospital one week ago and has had a fever for the past 3 days, is unable to lie flat due to a productive cough and has chills. The chest $\mathrm{x}$-ray has revealed the patient has community-acquired pneumonia and must be admitted to the hospital. You are asked to conduct a routine admission assessment, and these are your findings:

\section{Case progression}

K. F.'s vital signs are 158/84, HR 104, RR 32, T 103.4, 02 sats $86 \%$ on room air. You auscultate decreased breath sounds in the left lower lobe both anteriorly and posteriorly and hear course crackles in the Left upper lobe. You notice round rings and bruising on K.F.'s back. Her nails are dusky, she has a productive cough with rust-color sputum and is complaining of pain on her left side, and it is difficult for her to breathe. She is a non-drinker and nonsmoker and has been in good health up until now according to K. F's daughter. K.F.'s husband is deceased, she lives with her daughter who works. She appears well nourished, but there are rings and bruising on K.F.S back that are cause for concern. When the patient is asked about the marks on her back K.F. does not speak, she looks to her daughter. The daughter stated K.F. does not speak English and she is her mother's translator. The daughter stated, her mother was discharged from the hospital one week prior with oral medication for the same symptoms and was told to follow up with her provider. The daughter continued to explain her mother took Chinese herbs and treatments because they do not believe in antibiotics.

\section{Faculty participants responses}

Fifty percent of the faculty members voiced concern over the marks on the patient's back but did not ask the patient or daughter what they were. The other half of the faculty members were concerned with the readmission and wanted to know if the patient had pneumonia on the first admission, questioning if the patient took the medication with the Chinese herbs. Not one faculty member investigated what the patient and daughter were told or educated on for discharge.

\section{Case progression}

The doctor orders: regular diet, VS with temperature $\mathrm{q} 2 \mathrm{~h}$; maintenance IV of D5 $1 / 2 \mathrm{NS}$ at $125 \mathrm{ml} / \mathrm{hr}$; ceftriaxone $1 \mathrm{~g} \mathrm{IV}$ q24hx10, doxycycline $100 \mathrm{mg}$ bid x10; titrate oxygen to maintain oximeter $\mathrm{O}_{2}$ sats $>92 \%$; obtain sputum for culture and sensitivity x 3; draw blood cultures at 2 sites for temperature over $102 \mathrm{~F}$; CBC wit differential, basic metabolic panel, and urinalysis with C\&S as indicated; repeat chest X-ray in the morning. Naprosyn $250 \mathrm{mg}$ twice daily for temperature $>100.5 \mathrm{~F}$ and pain. You communicate what you observed on K.F.'s back to your charge nurse and want to call Elder Protective Services (EPS) to investigate K.F.'s home situation, that you feel she is being abused by her daughter.

\section{Faculty Participant Response}

One Hundred percent of the faculty utilized computers to research the marks on the patient's back.

\section{Case progression}

Explain what you have observed and what do you think is going on with this patient? How would you handle this situation? Should EPS be called to investigate what you observed to be abuse?

\section{Faculty participant response}

One hundred percent of the faculty stated they did not know anything about the Chinese culture and religious beliefs. Through open dialogue all faculty participants stated they would not call EPS and would talk to the daughter about this practice and to discuss the first admission and if the patient had taken the medication with the Chinese herbs. After further collaboration the faculty decided that the discharge plan did not include full education pertaining to the importance of taking the oral medication. Through computer research faculty learned about the Chinese culture of cupping that could be incorporated into the discharge plan but looking further into Chinese herbs and contraindications with the discharge medication would be a concern. More importantly the faculty themselves realized their lack of knowledge to cultural differences and it not being addressed enough in early nursing education. If the patient and daughter were explained the importance of completing the medication and possibly looking into the effects of the Chinese herbs with the medication maybe a better plan for the patient would have provided a safer outcome.

Three case studies similar to the one above consisted of a patient with various disease processes requiring personalized discharge planning and education for treatment at home. The participants evaluated the patient's and families' knowledge and ability to complete self-care and then were required to contact the appropriate interdisciplinary team members for assistance. The participants then formulated an individualized discharge planning and education plan for the patient. They worked in groups throughout this presentation utilizing a team-based learning approach that incorporated active learning. This learning strategy encourages collaboration along with strategies to promote critical thinking [8].

Participants were asked to research hospital readmission rates due to cultural competency and they did find several articles that concluded greater hospital cultural competency may improve overall patient experiences and contribute to general quality improvement to reduce readmission rates [9]. Another article faculty participants discovered was an article that revealed ethnic 
minority groups were at greater risk for unplanned readmission within a 30 day window of discharge and an excess length of stay [10]. This exercise brought awareness to the faculty participants the need to introduce culturally diverse discharge planning and education curriculum into early nursing school courses to better prepare nursing students to be culturally competent and not just culturally aware of their patients when they enter into the work force.

A variety of activities were utilized to accommodate the various learning styles of the students. A PowerPoint presentation will guide the participants through the activities. Participants at this level were made aware of these interdisciplinary team members and what their functions are. Computers were made available for participants to research the culture of the patient and their disease process. They were then provided the time to role play a scenario for culturally diverse discharge education and planning. The groups designated a patient, two nurses or a case manager or social worker, and possibly a family member which was dependent upon the size of the group. The curriculum was evidence-based and could easily be replicated by other schools of nursing programs by utilizing the same tools such as PowerPoint presentations, Prezi presentations, case studies, role play and/or simulation. These tools have minimal to no costs involved, which will not place a financial burden on the university or college. Nursing programs can also build upon each curriculum as the students' progress; therefore, upon graduation they will be able to apply the knowledge learned and utilize it in practice to potentially work towards positive patient outcomes and reduce readmission rates. The presentation took approximately one hour, and the development of the curriculum and presentation took this author approximately 12 weeks to complete.

\section{Analysis/Evaluation}

Based on a thorough web-based search of current evidencebased literature pertaining to this topic, there were several predisposing factor to this phenomenon. Poor discharge planning was the one expected influencing factor, but along with that came poor use of a standardized ISBAR tool, lack of communication among healthcare workers, lack of patient education, and cultural barriers. It is not just a national problem, but a global issue as well. Further research was conducted to investigate whether or not schools of nursing incorporate discharge planning into their curriculum. Articles researched discussed continuing education for graduates pertaining to discharge planning; however, there were no articles available that proved discharge planning was incorporated in the curriculum of the schools of nursing. Schools such as St. Luke's College of Nursing Research for the Development of Nursing Practice have held a continuing education class pertaining to discharge planning once a year since 2009 [11].

Through the literature review process, it was evident that this DNP project for discharge planning now needed to become the Culturally Diverse Discharge Education and Planning. The seriousness of this phenomenon has made this project facilitator extremely motivated to take this evidence and incorporate it into practice. To achieve the goal of this curriculum cultural discharge education and planning must be implemented in the curriculum of all schools of nursing. Originally this author was going to develop the curriculum and present this project to nursing students in the Fundamental Nursing course at Touro University Nevada. This research has now steered this author into another direction; to make an impact on the phenomenon presented, faculty of nursing schools need to be made aware of the prevalence of this issue. Dissemination of this evidence would have a greater impact if awareness were brought to faculty. Inviting faculty to the presentation of this project enlightened them to the ease of adding culturally diverse discharge education and planning into their nursing curriculum.

The project Culturally Diverse Discharge Planning and Education was presented on March 4, 2016 to eight nursing faculty members, one case manager, and one director of case management. Discussion topics were anonymously documented as a means of evaluation. Throughout the presentation, this project facilitator questioned the participants pertaining to the importance of discharge planning in a Fundamental Nursing course. After the introduction and a brief review of the literature, participants were asked if they felt culturally diverse discharge planning and education was a necessary topic for their nursing program; $100 \%$ (10) agreed it was. As the presentation case study unfolded, $90 \%$ (9) of the participants mentioned their lack of knowledge about the Chinese culture. They appreciated the opportunity to conduct research on-line, the Chinese culture pertaining to medicine. They worked as a team and $100 \%$ (10) mentioned they found the case study both educational and fun.

Each participant who attended and participated in the case study received one CEU. Therefore, as per the Nevada Board of Nursing each participant was required to answer four questions pertaining to the presentation. The questions were ranked using a Likert type scale from one to four, one being poor and four being excellent. The first question asked if the objectives were met and 100\% (10) stated yes and ranked the question with the number four. The second question asked if the speaker was effective, well-prepared and presented the material in an organized fashion and again $100 \%$ (10) ranked the question with a number four. The third question asked if the presentation increased the participants' knowledge pertaining to the topic $90 \%$ (9) stated yes and ranked the question with a number 4 (Excellent); 1\% (1) ranked the question with a number 3 (Good). The last question asked if the material presented is applicable to their area of practice and $100 \%$ (10) ranked the question with the number 4 (Excellent). During an informal verbal communication $100 \%$ (10) of the participants stated they were very interested in incorporating culturally diverse discharge planning and education into their curriculum. The case manager and the director of case management of UnitedHealthcare stated they would like to develop an internship collaboration with Touro University Nevada's School of Nursing in the future. The participants were very enthusiastic about the toolkit that was provided to them from the Agency for Healthcare Research and Quality. They all stated they realize that due to our diverse population there are cultural aspects of patient care that is not discussed at all at the bedside or in the community setting. This was an exercise to bring awareness of the need to start education and discharge planning in nursing at the educational level. Understanding the cultural needs of patients 
and better communication with all patients will reduce admissions to acute care facilities and readmission rates.

This author has started to disseminate the information from this project through a poster, presented at Touro University Nevada's Research Day on March 4, 2016. The information will again be presented at the Western Institute of Nursing (WIN) conference being held at the Disneyland Hotel in Anaheim, CA on April 6-9th, 2016.

The presentation can easily be replicated with no financial burden to the university. This author is planning to continue the development of courses for each level of nursing. After the curriculum is developed, this author is hopeful in having colleagues with research experience research the effectiveness of the curriculum for future publication.

\section{Dissemination}

This curriculum can easily be replicated with little to no cost for all schools of nursing. The American Association of Colleges of Nursing (2008) has a toolkit with detailed information pertaining to the development of culturally diverse discharge planning and education curriculum. Each participant was provided this toolkit to reference along with an invitation to call this presenter for assistance or a copy of the curriculum created for the Fundamental Nursing class.

This author was very excited that the participants accepted and embraced the curriculum Culturally Diverse Discharge Planning and Education. This author would like to publish the curriculum in the future to impact student outcomes and potentially reduce healthcare costs. Further studies would need to be performed to analyze the positive, negative, or null effect this curriculum may have. It would be a beneficial study this author can perform in conjunction with her practicum mentor who has research experience. This can be considered a demonstration of Ph.D. and DNP prepared educators working collaboratively to improve healthcare.

This author has had the opportunity to disseminate this DNP project on two occasions. The first time was at Touro University Nevada's research day. A poster was displayed for students and faculty members, and staff to view. Several students inquired about cultural diversity and the discharge process. Many instructors were interested on the impact of poor communication and readmission rates. The results of the project were discussed and most of the inquiring instructors stated they would like to incorporate culturally diverse discharge planning and education into their curriculum. Toolkits were offered to instructors.

The second time this project was disseminated was at the Western Institute of Nursing Conference in California. The poster was displayed for attendees to view. Several Ph.D. instructors were very interested in the project, provided me their cards, and asked if this author would send the project to them once it is published.

\section{Acknowledgement}

\section{None.}

\section{Conflict of Interest}

No conflict of interest.

\section{References}

1. Kocher RP, Adashi EY (2011) Hospital readmissions and the Affordable Care Act paying for coordinated quality care. The Journal of the American Medical Association 306 (16): 1794-1795.

2. Wrobleski DS, Joswiak ME, Dunn DF, Maxson PM, Holland DE (2014) Discharge planning rounds to the bedside: A patient-and family centered approach. MEDSURG Nursing, 23 (2): 111-116.

3. Weil AR (2015) Hospital costs and quality.

4. Wong EL, Yam CH, Cheung AW, Leung MC, Chan FW, et al (2011) Barriers to effective discharge planning: a qualitative study investigating the perspectives of frontline healthcare professionals. BMC Health Services Research 11(1): 242.

5. Leininger MM, McFarland MR (2010) Madeleine Leininger's theory of culture care diversity and universality. In ME Parker \& MC Smith (Eds.) Nursing theories \& nursing practice ( $3^{\text {rd }}$ edn), Philadelphia, F.A. Davis Company, USA, pp. 317-336.

6. Buyayisqui M, Bordoni N, Garbossa G (2013) Overcoming language and cultural barriers: A graphical communication tool to perform a parasitological screening in two vulnerable populations from Argentina. Journal of Health Communication 18(1): 92-104.

7. Corso P, Taylor N, Bennett J, Ingels J, Self-Brown S, et al. (2014) Marginal cost analysis of two Train-the Trainer models for implementing safe care. Western Journal of Emergency Medicine: Integrating Emergency Care with Population Health 15(5): 623-626.

8. Rowles CJ (2012) Strategies to promote critical thinking and active learning. In D. M. Billings \& J. A. Halstead (Eds.), Teaching in nursing: A guide for faculty ( $4^{\text {th }}$ edn), St. Louis, Elsevier, Netherlands, pp. 258-284.

9. Welch Maldonado R, Elliot MN, Pradhan R, Schiller C, Hall A, et al. (2012). Can hospital cultural competency reduce disparities in patient experiences with care. Med Care 50 (0): 48-55.

10. De Bruijne MC, Van Rosse F, Uiters E, Droomers M, Suurmond J, et al (2013) Ethnic variations in unplanned readmissions and excess length of hospital stay: A nationwide record linked to cohort study. European Journal of Public Health 23 (6): 964-971.

11. Tashiro M, Yamada M, Utsunomiya H, Yoshida C, Nagae H, et al. (2013) Exchange gathering for nursing graduates of the discharge planning continuing education program. Bulletin of St. Luke's College of Nursing 39: 61-64.

12. American Association of Colleges of Nursing (2015) About leadership networks.

13. Novak K, Fairchild R (2012) Bedside reporting and SBAR: Improving patient communication and satisfaction. Journal of Pediatric Nursing 27(6): 760-762.

14. Centers for Disease Control and Prevention (2015) Health expenditures.

15. Centers for Medicare \& Medicaid Services (2014) Readmissions reduction program.

16. Collaborative Institutional Training Initiative (2013) Privacy and confidentiality.

17. Compton J, Copeland K, Flanders S, Cassity C, Spetman M, et al. (2012). Implementing SBAR across a large multihospital health system. Joint Commission Journal on Quality \& Patient Safety 38(6):261-268.

18. Gordon MD (2012) Bedside reporting and SBAR: Improving patient communication and satisfaction. Journal of Pediatric Nursing 27(6): 760-762.

19. Harkess L, Kaddoura M (2015) Culture and cultural competence in nursing education and practice: The state of the art. Nursing Forum 51(3):211-22.

20. Mc Gee P, Johnson MR (2014) Developing cultural competence in palliative care. British Journal of Community Nursing19(3): 91-93. 
21. Moran K (2014) The proposal. In: Moran K, Burson R and Conrad D (Eds.), The Doctor of Nursing practice scholarly project: A framework for success Burlington, Jones \& Bartlett Learning, USA, pp. 239-275.

22. Murphy JC, Carson Warner CO. Train-the-trainer manual: Mentoring adult learners.
23. Reddick B, Holland C (2015) Reinforcing discharge education and planning. Nursing Management 46(5): 10-14.

24. Reyes H, Hadley L, Davenport D (2013) A comparative analysis of cultural competence in beginning and graduating nursing students. International Scholarly Research Network 1-5. 